\title{
Pulsed Electromagnetic Field Stimulators Efficacy for Noninvasive Bone Growth in Spine Surgery
}

\author{
Brian Fiani, ${ }^{1}$ Athanasios Kondilis, ${ }^{2}$ Juliana Runnels, ${ }^{3}$ Preston Rippe, ${ }^{4}$ Cyrus Davati ${ }^{5}$ \\ Department of Neurosurgery,' Desert Regional Medical Center, Palm Springs, CA, USA \\ Michigan State University College of Osteopathic Medicine, ${ }^{2}$ East Lansing, MI, USA \\ School of Medicine, University of New Mexico, ${ }^{3}$ Albuquerque, NM, USA \\ University of Pikeville-Kentucky College of Osteopathic Medicine, ${ }^{4}$ Pikeville, KY, USA \\ New York Institute of Technology College of Osteopathic Medicine, ${ }^{5}$ Old Westbury, NY, USA
}

The growth of pulsed electromagnetic field (PEMF) therapy and its progress over the years for use in post-operative bone growth has been revolutionary in its effect on bone tissue proliferation and vascular flow. However, further progress in PEMF therapy has been difficult due to lack of more evidence-based understanding of its mechanism of action. Our objective was to review the current understanding of bone growth physiology, the mechanism of PEMF therapy action along with its application in spinal surgery and associated outcomes. The authors of this review examined multiple controlled, comparative, and cohort studies to compare fusion rates of patients undergoing PEMF stimulation. Examining spinal fusion rates, a rounded comparison of post-fusion outcomes with and without bone stimulator was performed. Results showed that postoperative spinal surgery PEMF stimulation had higher rates of fusion than control groups. Though PEMF therapy was proven more effective, multiple factors contributed to difficulty in patient compliance for use. Extended timeframe of treatment and cost of treatment were the main obstacles to full compliance. This review showed that PEMF therapy presented an increased rate of recovery in patients, supporting the use of these devices as an effective post-surgical aid. Given the recent advances in the development of PEMF devices, affordability and access will be much easier suited to the patient population, allowing for more readily available treatment options.

Key Words : Bone stimulator · Bone growth · Pulsed electromagnetic field · Spinal fusion · Ossification.

\section{INTRODUCTION}

Pulsed electromagnetic field (PEMF) therapy is a noninvasive post-operative technique that stimulates and facilitates healing by directing magnetic fields through tissue, utilizing the movement of electrons to affect the cellular physiology of excitable cells ${ }^{45}$. The use of low frequency signals was first in- troduced as a new approach for treatment of non-union fractures in the 1970's by Andrew Bassett ${ }^{31}$. While only Food and Drug Administration (FDA) approved for application in the USA for non-union fractures, pulsed radiofrequency electromagnetic fields later became FDA approved for use of treatment of pain and edema in superficial soft tissues ${ }^{31}$. PEMF therapy has displayed significant effects on excitable and non-

- Received : September 11, 2020 •Accepted : October 29, 2020

- Address for reprints : Brian Fiani

Department of Neurosurgery, Desert Regional Medical Center, 1150 N. Indian Canyon Dr., Palm Springs, CA 92262, USA

Tel : +1-760-416-4511, Fax : +1-760-323-6201, E-mail : bfiani@outlook.com, ORCID : https://orcid.org/0000-0003-1700-4633

This is an Open Access article distributed under the terms of the Creative Commons Attribution Non-Commercial License (http://creativecommons.org/licenses/by-nc/4.0) which permits unrestricted non-commercial use, distribution, and reproduction in any medium, provided the original work is properly cited. 
excitable cells leading to osteogenesis and chondrocyte proliferation $^{45)}$. It is now accepted that PEMF therapy is capable of initiating healing processes of non-union fractures, pain relief, multiple sclerosis, and Parkinson's disease ${ }^{31)}$.

Spinal arthrodesis, or spinal fusion, is one of the most common surgical procedures for the treatment of various spinal pathologies, with aim to achieve bone fusion at a mobile segment by the transplantation of autologous, allogenic, or artificial bone graft to induce bone modeling and remodeling ${ }^{29)}$. Pseudoarthrosis, the failure of union to develop after fusion, can occur in $5-35 \%$ of patients ${ }^{38,40)}$. Failure of union can cause loss of correction and instrumentation failure or deterioration of patients' quality of life, expressing the need for early and successful bone fusion to provide better clinical outcomes ${ }^{29)}$. PEMF post-operative therapy can potentially be used to achieve such success following spinal fusions to decrease recovery time and reduce pain ${ }^{40)}$. PEMF therapy also includes benefits such as ease of use, relatively low cost, and unique ability to be brought into patient's homes without requiring frequent clinic visits. Additionally, use of PEMF post-operatively does not interfere with surgical techniques or standard rehabilitation protocols ${ }^{24)}$. Only Class III electromagnetic field devices designed by Orthofix Inc., and approved by the FDA, reside in the category of bone growth simulation/osteogenesis stimulation : Physio-Stim, Spinal-Stim, and Cervical-Stim ${ }^{46}$. Since electromagnetic therapy FDA approval, PEMF therapy has been used primarily for non-union fractures as well as for enhancement of bone formation after lumbar and cervical spine fusion surgery ${ }^{24)}$.

\section{BONE GROWTH PHYSIOLOGY}

The skeletal components of the vertebral column are formed by endochondral ossification, the formation of cartilage from mesenchymal cells and replacement of that cartilage with bone tissue. In the primary ossification center, mesenchymal cells express transcription factors Paxl and Scleraxis, activating cartilage-specific genes, and further condense into nodules that differentiate into chondrocytes. Chondrocytes divide rapidly, secreting cartilage-specific extracellular matrix, and increase in volume to become hypertrophic chondrocytes that become the model for bone. The now large chondrocytes can produce a specialized matrix that can become mineral- ized by calcium carbonate. Finally, surrounding blood vessels infiltrate, hypertrophic chondrocytes die, and the space becomes bone marrow. Groups of cells that surround the cartilage differentiate into osteoblasts, cells that begin to form the bone matrix on the degraded cartilage. Eventually, the cartilage is replaced by bone and the endochondral ossification spreads outwards from the center of the bone ${ }^{18)}$. With the exception of coccygeal vertebrae, there are three ossification centers per vertebra : one in its body and one in either neural arch. The first vertebral body ossification centers in the cervical spine begin at week eight of development and progresses caudad; ossification centers in the lower thoracic vertebrae and first lumbar vertebrae occur at week ten of development and progresses cephalad and caudad ${ }^{5}$. Secondary ossification centers near the ends of the bones form and push out the cartilaginous ends of the bone, called epiphyseal growth plates. This allows bones to grow after birth ${ }^{18)}$. Unlike long bones, however, vertebral body epiphyses never ossify and after the end of the growth period of life they are reduced to thin cartilage called hyaline cartilage, sitting between the vertebral body and intervertebral disc ${ }^{14)}$. After primary bone is completely formed, the bones of the human skeleton constantly undergo bone modeling during life in order to adapt to changing biomechanical forces. Bone also undergoes remodeling in order to replace old, microdamaged bone with newer bone that increases mechanical strength and preserves overall bone strength. Remodeling sites develop in a random manner, but are also targeted to areas needing extra repair ${ }^{8)}$.

Spinal fusion procedures utilize a scaffold that undergo specialized stages for proper bony fusion : inflammation, vascularization, osteoinduction, osteoconduction, and remodeling. This process includes primary membranous bone to form near decorticated bone, endochondral ossification, osteoblast/ osteoclast differentiation, in-growth into host bone, and remodeling to increase cortical rim thickness around fusion ${ }^{21)}$. The process of spinal fusion bone growth relies primarily on the remodeling process of bone. At bone grafted sites in posterolateral fusions, membranous bone formation begins primarily, and increased volume of woven bone and endochondral ossification can be seen. Interbody fusion, however, is more demanding biologically as compared to posterolateral fusion due to the hypo-vascular and low nutrient condition of the intervertebral space ${ }^{29)}$. Bone metabolism is constant balance between osteoblast and osteoclast activity, which is regu- 
lated by metabolic, endocrine, and mechanical signaling pathways ${ }^{29)}$. Osteoclasts are giant multinucleated cells that originate from hematopoietic cells in the bone marrow, responsible for resorption of the calcified matrix of bone through secretion of acids and collagenolytic enzymes. Differentiation and activation of osteoclasts are dependent on the receptor activator of nuclear factor kB ligand (RANKL) produced by osteoblasts, while osteoclast inactivation is controlled in part by osteoprotegerin (OPG), a soluble RANKL decoy that inhibits osteoclastogenesis by RANKL-RANKOPG interaction. Therefore, RANKL and OPG play significant roles in bone homeostasis through osteoclast regulation $^{29)}$.

Bone fusion following spinal arthrodesis can be achieved when the balance of bone homeostasis is shifted towards increased bone formation by the upregulation of osteoblast activity, as well as regulation of biological agents that promote osteoblast activity ${ }^{29)}$. Osteoblast differentiation is stimulated by local factors such as bisphosphonates and bone morphogenetic proteins (BMPs), specifically BMP2 and BMP4. Systemic signals such as Leptin, parathyroid hormone, growth hormone, or insulin-like growth factor 1 , and sex hormones play significant roles in upregulation of osteoplastic lineage cell development ${ }^{29)}$. Factors that may hinder fusion of vertebrae include long-term use of non-steroidal anti-inflammatory agents and ${ }^{38)}$.

\section{PEMF GENERATORS}

For years, PEMF stimulation has aided in accelerating the healing process of spinal fusions and fracture healing. In order to understand the mechanism of action, the electrophysiology of bone tissue must be first accounted for. Broken bone tissue creates an electrical charge. Bone tissue deposits in regions of negative charge and is absorbed in areas of positive charge ${ }^{30}$. PEMF works by inducing negative charge at the fracture site, thus stimulating further tissue deposition at that location. PEMF works at the molecular, cellular, and tissue level.

On the molecular level, PEMF works by stimulating an electrical impulse across the cell membrane of osteoblasts, inducing an intracellular response that activates signaling pathways immediately. PEMF works by increasing calcium binding to calmodulin through $\mathrm{Ca} / \mathrm{CaM}$ pathway ${ }^{42}$. The Ca-Cam com- plex binds to nitric oxide synthase, catalyzing the release of nitric oxide. Within seconds, anti-inflammatory effects are initiated, with blood and lymph flow increased. Pain and edema starts to decrease within minutes. The NO release causes conversion of guanosine-5'-triphosphate to cyclic guanosine monophosphate, stimulating growth factors vascular endothelial growth factor for angiogenesis and tumor necrosis factor- $\alpha$ for collagen accumulation and granulation ${ }^{42}$.

PEMF has varying benefits through the several phases of bone healing. During initial hematoma formation and angiogenesis, PEMF stimulates increased blood vessel production. During the second phase of soft callus formation and mineralization of newly formed bone matrix, PEMF stimulates the calcium influx that activates the signal transduction pathways and thus growth factor release ${ }^{42}$. Once the hard callus begins to mineralize, PEMF further calcifies the fibrocartilage. During the final remodeling phase of lamellar bone formation, PEMF increases osteoblast deposition rate, thus solidifying the remodeled bone.

One of the biggest factors in correctly engineering PEMF devices is proper diagnostics of precise dosage of EMF that the target location must receive. Several methods of biophysical dosimetry have developed, including myosin phosphorylation assay that predicts the efficiency of EMF signals ${ }^{30)}$. The same signal may have different efficiency depending on the particular target and medical problem being treated ${ }^{30)}$. PEMF signals in clinical use have a variety of designs, however the most widely used wave shape is the sine wave with $60 \mathrm{~Hz}$ frequency in North America and $50 \mathrm{~Hz}$ in the rest of the world ${ }^{30)}$. The first approved EMF signal for therapeutic application uses the pulse burst approach : an asymmetrical signal with a 15 burst per second repetition rate ${ }^{30)}$. This signal has proved successful in use of healing nonunion and delayed bone fractures ${ }^{30}$.

\section{INDICATIONS}

Due to the ramifications of improper bone healing of the spine affecting musculoskeletal integrity and progressing to further damage to the bone, it is imperative to determine the indications for PEMF stimulation ${ }^{13)}$. However, not all stimulators are equally effective in all situations and therefore certain stimulators should be prioritized depending on a patient's clinical presentation. Regardless, PEMF stimulators are an 
ideal non-invasive option for patients as an adjunct to spinal surgery as these devices can be worn between 3-8 hours daily for 3-6 months postoperatively; made even more effective as these devices can be fitted into a post-operative brace ${ }^{26)}$.

One such device is offered by manufacturer Zimmer Biom$\mathrm{et}^{50)}$. The Biomet ${ }^{\oplus} \mathrm{EBI}$ bone healing system is primarily indicated in cases of fracture non-union, as well as failed fusions and appendicular congenital pseudoarthrosis ${ }^{16}$. For lumbar fusions, the Biomet ${ }^{\oplus}$ SpinalPak ${ }^{\oplus}$ is indicated due to its increased rate of healing compared to placebo ${ }^{199}$. Another set of devices is offered by Orthofix; the first of which is the Cervical-Stim Osteogenesis Stimulator ${ }^{\left.\mathrm{TM}{ }^{36}\right)}$. This device is indicated in cases of cervical spine fusion as an adjunct to surgery in patients at high risk for non-fusion. The Spinal-Stim Osteogenesis Stimulator ${ }^{\mathrm{TM}}$ is used either as an adjunct to spinal fusion surgery or treating a previously failed fusion (Simmons et al. $1985)^{33,34)}$. The additional benefit here involves $360^{\circ}$ of coverage at the fusion site and treatment of up to 5 vertebral levels with a single device ${ }^{49)}$. A third device from Orthofix is the Physio-Stim Osteogenesis Stimulator ${ }^{\mathrm{TM}}$. Indications for this device includes established nonunion secondary to trauma, assuming such a defect is less than one-half the width of the bone to be treated ${ }^{17)}$. As a result of prior clinical studies, we find that PEMF stimulators provide an effective adjunct to successful or failed spinal fusion surgery by reducing recovery time and incidences of pseudoarthroses ${ }^{20)}$.

\section{NOVEL USES}

The greatest benefits of PEMF therapy have been proven for those suffering traumatic wounds, fractures, and inherited musculoskeletal disorders. Due to the electrical charge in cell tissue, EMF signals allow cellular migration to the wound area, thus preserving metabolic conditions. Current public interest in EMF lies in furthering the aid of alleviating pain. Recent advances in PEMF therapy suggest that certain magnetic fields aid in treatment of Parkinson's, Alzheimer, and Reflex sympathetic disorders where current medical protocol has done so little ${ }^{30)}$. PEMF therapy has also been beneficial in treatment of chronic pain associated with connective tissue injury and joint-associated soft tissue injury.

Beyond its use in anti-inflammatory mediation and cell proliferation, PEMF therapy has broadened to different spe- cialties like plastic surgery. PEMF has been used as a dual coil applicator for breast surgery, and as a single coil used for pain relief after abdominoplasty ${ }^{42)}$. Clinical results show that postoperative pain and edema disseminates quickly and patients are generally ready for discharge the first day out of surgery ${ }^{42}$. In the field of Otolaryngology, PEMF therapy has proven beneficial in its ability to enhance circulation and increase vascular flow in nasal defect surgery. Another novel use in PEMF therapy is its use of the $\mathrm{Ca} / \mathrm{CaM} / \mathrm{NO}$ pathway for treating chronic non-healing wounds. Chronically opened wounds begin closing between 6 to 10 weeks of this treatment ${ }^{42}$.

\section{LIMITATIONS}

A misconception of PEMF therapy is that the patients must understand that it is a complementary therapy in addition to their current post-operative regiment. Post-operative recovery measures consist of diet and pharmaceutical intervention that must be compliantly kept up with on the patient's end, and therefore PEMF therapy alone will not be responsible in the healing process if other additive measures are not continuously adhered to.

One main limitation of PEMF therapy is its ability to induce oxidative stress. Due to increasing blood flow and circulation induced by nitric oxide release, oxygen radicals accumulate and cause harmful effects on the body ${ }^{47}$. Increased oxidative stress causes cell damage and accelerates development of various disease conditions including Alzheimer's, cancer, and diabetes ${ }^{47)}$. Patients are advised to eat foods high in antioxidants to counteract these systemic effects. A factor in consideration is its effects on nerve stimulation. Due to nerve cell stimulation, increased circulation and signal traffic downstream of the nerve may exacerbate the pain. This commonly occurs in patients suffering from previous fractures, in which magnetic therapy stimulation not only causes pain at the current fracture site, but also at a distal location that once suffered scarring ${ }^{45}$. In some instances, electromagnetic therapy may stimulate pain in a random location that normally doesn't experience discomfort. This may indicate an underlying issue and possible internal scarring a patient is unaware of. Another limitation of PEMF therapy is its ability to lower blood pressure and decrease heart rate ${ }^{39)}$. This can pose risks for elderly patients on blood-pressure-lowering medications, patients with decreased vascular 
reflexes, and immunocompromised patients with systemic infections ${ }^{39)}$. Many of these patients can faint when rising from a seated position, so safety concerns must be addressed before placing these patients on PEMF therapy.

\section{OUTCOMES}

The primary aim of PEMF stimulation after spinal surgery is to facilitate arthrodesis. Arthrodesis, also referred to as fusion, describes the artificial induction of ossification to join two bones. Successful spinal fusion can be defined radiographically or clinically. The authors of this review identified four randomized controlled trials (RCTs) ${ }^{15,25,28,33)}$, two retro- spective comparative studies ${ }^{9,32)}$, two retrospective cohort studies ${ }^{7,12)}$, and one case series ${ }^{41)}$ that compared fusion rates of patients who received PEMF stimulation therapy and controls. The nine included studies are presented in Table 1. Corresponding fusion rates among the six studies designed to include treatment and control groups are represented by the graph in Fig. 1. The level of evidence reported by each study was assessed using the GRADES criteria ${ }^{4)}$. In all studies, fusion was confirmed using radiographic criteria, and patients were evaluated for solid arthrodesis 9-16 months after spinal surgery.

The rate of fusion reported by all studies assessing PEMF stimulation ranged from 64-98\%. Patients treated with PEMF stimulation as an adjunct to spinal surgery had higher rates of

Table 1. Select publications reporting fusion rates in patients treated with PEMF stimulators as an adjunct to spinal surgery.

\begin{tabular}{|c|c|c|c|c|c|c|}
\hline Study & $\mathrm{LOI}^{*}$ & Procedure & F/U (months) & PEMF fusion & Control fusion & $p$-value \\
\hline Coric et al. ${ }^{9)}$ (2018) & III & ACDF & 12 & $92.6 \%(201 / 217)$ & $82.6 \%(76 / 92)$ & $<0.05$ \\
\hline Foley et al. ${ }^{15)}$ (2008) & $\|$ & ACDF & 12 & $92.8 \%(116 / 125)$ & $86.7 \%(104 / 120)$ & $>0.05$ \\
\hline Simmons et al. ${ }^{41)}$ (2004) & IV & LIF & 12 & $67 \%(67 / 100)$ & - & - \\
\hline Linovitz et al. ${ }^{28)}(2002)$ & 1 & LIF & 9 & $64 \%(66 / 104)$ & $43 \%(48 / 97)$ & $>0.05$ \\
\hline Bose $^{7)}(2001)$ & IV & LIF & 16 & $98 \%(47 / 48)$ & - & - \\
\hline Jenis et al. ${ }^{25)}$ (2000) & $\|$ & LIF & 12 & $59 \%(10 / 17)$ & $82 \%(18 / 22)$ & $>0.05$ \\
\hline $\operatorname{Marks}^{32)}(2000)$ & III & LIF & 16 & $97.6 \%(41 / 42)$ & $52.6 \%(10 / 19)$ & $<0.001$ \\
\hline Mooney $^{33)}(1990)$ & $\|$ & LIF & 12 & $92.2 \%(59 / 64)$ & $67.9 \%(36 / 53)$ & $<0.005$ \\
\hline Di Silvestre and Savini $i^{12)}$ (1992) & IV & LIF & 12 & $96 \%(30 / 31)$ & - & - \\
\hline
\end{tabular}

*Level of evidence was determined using GRADE criteria. PEMP : pulsed electromagnetic field, LOI : level of evidence, F/U : follow up in months, ACDF : anterior cervical discectomy and fusion, LIF : lumbar interbody fusion

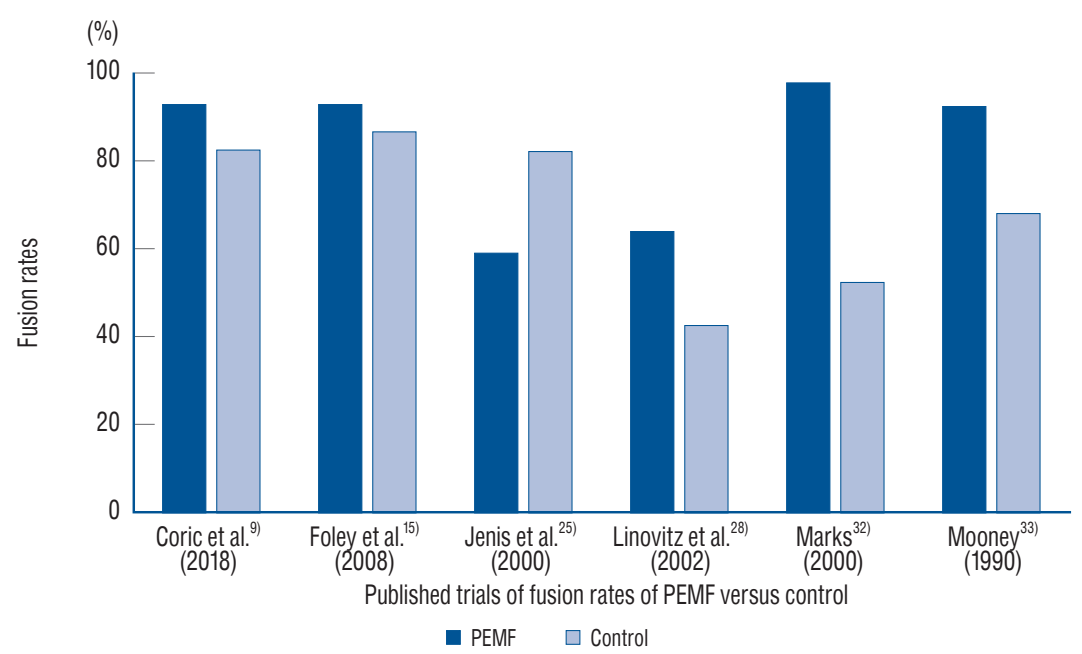

Fig. 1. Fusion rates reported among patients treated with PEMF versus controls. PEMP : pulsed electromagnetic field. 
fusion than sham, placebo, or no stimulation control groups. Among studies comparing this therapy to a control group, fusion rates ranged from 64-97.6\% with PEMF stimulation and $43-86.7 \%$ for controls. The results of our review are consistent with three previous meta-analyses. Akhter et al. ${ }^{3)}$ published a recent meta-analysis of the RCTs included in this review and found the odds ratio of successful fusion with PEMF stimulation relative to control was 2.88 (95\% confidence interval [CI], $1.18-7.04 ; p=0.02)$. Similarly, in a meta-analysis by Cottrill et al. ${ }^{10)}$ the odds ratio was 2.45 (95\% CI, 1.20-4.99) and therefore authors concluded that patients who received PEMF stimulator therapy had significant improvements in fusion rate relative to controls ( $p=0.014)$. In a meta-analysis by Tian et al. ${ }^{43}$, the pooled fusion rate in PEMF clinical studies was 85\% (95\% CI, 76-91\%).

The PEMF stimulator device can be cumbersome for patients to use, and thus compliance represents a potential barrier to effective treatment. Patients must wear the PEMF stimulation device for approximately 8 hours each day ${ }^{3}$. When used post-operatively, the overall treatment duration can range from 3-9 months ${ }^{27}$. In the double-blinded RCT by Mooney ${ }^{33)}$, less than $80 \%$ of the original cohort was included in the study due to noncompliance.

\section{DISCUSSION WITH PATIENTS}

Regarding the patient, questions must be dealt with in order to alleviate concerns about use and function of PEMF devices. A primary concern with patients involves invasiveness of the treatment. Unlike implantable DC electrical stimulators or semi-invasive percutaneous cathode stimulators, PEMF devices are worn externally in an entirely non-invasive fashion and in many cases can be fitted into a post-surgical brace ${ }^{26)}$. In all cases, the devices must be positioned so that the electromagnetic coils overlie the region to be treated, while the signal generator position and strength of the electromagnetic field will vary based on device model and patient comfort; all of which can be adjusted accordingly. The electrical current through the coils will generate a time-varying magnetic field which induces an electric field within the tissue, stimulating bone growth ${ }^{27}$. The strength of the electromagnetic field can vary based on the device parameters. The most commonly used PEMF devices come from medical equipment manufac- turers Zimmer Biomet (particularly their EBI and SpinalPak bone healing systems) and Orthofix (including the PhysioStim, Cervical-Stim, and Spinal-Stim Osteogenesis Stimulators). Indications for the specific devices are described in detail in the "Indications" section.

Another concern involves timeframe of treatment. Typically, a surgeon will prescribe a PEMF device to be worn between 3-8 hours daily for 3-9 months post-operatively depending on the severity of injury and type of stimulator used ${ }^{34)}$. It is imperative that the surgeon educates the patient on the importance of adhering to the treatment regimen and charging and maintaining their device to decrease rates of non-compliance. To alleviate issues with non-compliance, both manufacturers Zimmer Biomet and Orthofix supply smartphone applications for the physician to remotely track patient adherence to treatment allowing the physician flexibility to tailor the treatment schedule to a patient's daily routine and level of commitment to said treatment. Patients with additional questions are supplied with contact information for troubleshooting, maintenance, and general device concerns from both manufacturers ${ }^{37,50)}$. Incidences of broken devices are also directly dealt with by the manufacturer for their specific device.

Ultimately, the main concern dealt with patients involving a PEMF device involves cost of treatment. Treatment cost of PEMF devices can range from $\$ 500$ and can increase substantially to over $\$ 3000^{22,35)}$. Further, insurance coverage varies between patients, with roughly $72 \%$ of respondents to a Huang et al. ${ }^{23)}$ study in 2004 having formal coverage for bone stimulator. However, recent policy changes and expanded coverage allow a physician greater freedom in prescribing PEMF devices to patients assuming the patient meets the criteria for coverage and reimbursement, which includes the aforementioned indications ${ }^{2,6,11)}$. As a result, it is imperative patients and physicians properly discuss the level to which insurance will cover treatment costs as these policies are regularly changed and updated. When analyzing costs of treatment; indirect, direct, intangible and future healthcare expenses must all be factored in for the patient. Particularly in cases of nonunion fracture healing, PEMF devices are associated with significantly lower total healthcare and predicted future healthcare-related costs and increased rates of recovery compared to unstimulated treatment or a low intensity pulsed ultrasound stimulator counterpart (LIPUS) ${ }^{48)}$. Wu et al. ${ }^{48)}$ determined in a sample of 
11628 patients that predicted future healthcare costs for patients using a PEMF device was $\$ 9100$ compared to $\$ 10255$ and $\$ 10354$ for LIPUS and non-stimulatory treatment respectively, supporting the notion for these devices as a medically beneficial as well as a cost-effective adjunct to surgery.

\section{CONCLUSION}

In this paper, we presented a comprehensive overview of PEMF stimulation and its efficacy in spinal fusion. These highly portable devices have demonstrated decreased longterm healthcare costs and increased rates of recovery in patients supporting the notion for these devices as an effective adjunct to surgery. Issues may arise with patient adherence due to the length of time required for treatment, and future research and development will hopefully explore methods of stimulation that do not require extended periods of wear throughout the day and decreasing the total length of treatment for full recovery. It is imperative that future studies also investigate the exact mechanism of action as to how these bone stimulators increase the rate of healing as there are conflicting reports that implicate extracellular matrix proteins, growth factor and protein synthesis, increased vascular flow, osteoblastic proliferation, transmembrane receptor upregulation, or reduction in inflammatory markers as potential targets of PEMF stimulation ${ }^{1,44)}$. Determining a primary mechanism of action is imperative for potential future therapeutics to potentially augment the healing process promoted by PEMF devices in order to address patient concerns involving recovery rate and length of treatment. Regardless, the current state of the literature supports the continued use and further investigation of PEMF devices as an effective adjunct for promoting bone growth and recovery following spinal surgery.

\section{CONFLICTS OF INTEREST}

No potential conflict of interest relevant to this article was reported.

\section{INFORMED CONSENT}

This type of study does not require informed consent.

\section{AUTHOR CONTRIBUTIONS}

\author{
Conceptualization : BF \\ Data curation : $\mathrm{BF}$ \\ Formal analysis : BF \\ Methodology : BF \\ Project administration : $\mathrm{BF}$ \\ Visualization : BF \\ Writing - original draft : $\mathrm{BF}, \mathrm{AK}, \mathrm{JR}, \mathrm{PR}, \mathrm{CD}$ \\ Writing - review \& editing : $\mathrm{BF}$
}

\section{ORCID}

Brian Fiani https://orcid.org/0000-0003-1700-4633

Athanasios Kondilis https://orcid.org/0000-0003-2349-2821

Juliana Runnels https://orcid.org/0000-0003-4535-2766

Preston Rippe ～https://orcid.org/0000-0002-9610-153X

Cyrus Davati https://orcid.org/0000-0001-6155-2392

\section{References}

1. Aaron RK, Ciombor DM, Simon BJ : Treatment of nonunions with electric and electromagnetic fields. Clin Orthop Relat Res (419) : 21-29, 2004

2. Aetna Inc. : High-frequency pulsed electromagnetic stimulation. Available at : http://www.aetna.com/cpb/medical/data/100_199/0175. html

3. Akhter S, Qureshi AR, Aleem I, El-Khechen HA, Khan S, Sikder O, et al. : Efficacy of electrical stimulation for spinal fusion: a systematic review and meta-analysis of randomized controlled trials. Sci Rep $10: 4568$, 2020

4. Atkins $D$, Best $D$, Briss PA, Eccles M, Falck-Ytter $Y$, Flottorp $S$, et al. : Grading quality of evidence and strength of recommendations. BMJ 328 : 1490, 2004

5. Baumgart M, Wiśniewski M, Grzonkowska M, Małkowski B, Badura M, Dąbrowska $M$, et al. : Digital image analysis of ossification centers in the axial dens and body in the human fetus. Surg Radiol Anat 38 : 11951203, 2016

6. Blue Cross Blue Shield of Massachusetts : Electrical Bone Growth Stimulation of the Appendicular Skeleton. Available at : https:// 
www.bluecrossma.org/medical-policies/sites/g/files/csphws2091/files/ acquiadam-assets/499\%20Electrical\%20Bone\%20Growth\%20Stimulation\%20of\%20the\%20Appendicular\%20Skeleton\%20prn.pdf

7. Bose $B$ : Outcomes after posterolateral lumbar fusion with instrumentation in patients treated with adjunctive pulsed electromagnetic field stimulation. Adv Ther 18 : 12-20, 2001

8. Clarke B : Normal bone anatomy and physiology. Clin J Am Soc Nephrol 3 Suppl 3(Suppl 3) : S131-S139, 2008

9. Coric D, Bullard DE, Patel VV, Ryaby JT, Atkinson BL, He D, et al. : Pulsed electromagnetic field stimulation may improve fusion rates in cervical arthrodesis in high-risk populations. Bone Joint Res 7 : 124-130, 2018

10. Cottrill E, Pennington Z, Ahmed AK, Lubelski D, Goodwin ML, PerdomoPantoja A, et al. : The effect of electrical stimulation therapies on spinal fusion: a cross-disciplinary systematic review and meta-analysis of the preclinical and clinical data. J Neurosurg Spine 32 : 106-126, 2020

11. CPT Only - American Medical Association : Noninvasive electrical bone growth stimulation of the appendicular skeleton. Available at : https://www.anthem.com/dam/medpolicies/abc/active/guidelines/gl_pw_d055254.html

12. Di Silvestre M, Savini R : Pulsing electromagnetic fields (PEMFs) in spinal fusion: preliminary clinical results. Chir Organi Mov 77 : 289-294, 1992

13. Dimitriou $R$, Jones $E$, McGonagle $D$, Giannoudis $P V$ : Bone regeneration: current concepts and future directions. BMC Med $9: 66,2011$

14. Doskocil M, Valouch P, Pazderka V : On vertebral body growth. Funct Dev Morphol 3 : 149-155, 1993

15. Foley KT, Mroz TE, Arnold PM, Chandler HC Jr, Dixon RA, Girasole GJ, et al. : Randomized, prospective, and controlled clinical trial of pulsed electromagnetic field stimulation for cervical fusion. Spine J 8 : 436442, 2008

16. Frykman GK, Taleisnik J, Peters G, Kaufman R, Helal B, Wood VE, et al. : Treatment of nonunited scaphoid fractures by pulsed electromagnetic field and cast. J Hand Surg Am 11 : 344-349, 1986

17. Garland DE, Moses B, Salyer W : Long-term follow-up of fracture nonunions treated with PEMFs. Contemp Orthop 22 : 295-302, 1991

18. Gilbert SF : Developmental Biology, ed 6. Sunderland : Sinauer Associates, 2000

19. Goodwin $C B$, Brighton $C T$, Guyer $R D$, Johnson JR, Light $K I$, Yuan $H A$ : $A$ double-blind study of capacitively coupled electrical stimulation as an adjunct to lumbar spinal fusions. Spine (Phila Pa 1976) 24 : 13491356; discussion 1357, 1999

20. Griffin $M$, Bayat $A$ : Electrical stimulation in bone healing: critical analysis by evaluating levels of evidence. Eplasty 11 : e34, 2011

21. Gupta A, Kukkar N, Sharif K, Main BJ, Albers CE, El-Amin lii SF : Bone graft substitutes for spine fusion: a brief review. World J Orthop 6 : 449-456, 2015

22. Hannemann PF, Essers BA, Schots JP, Dullaert K, Poeze M, Brink PR : Functional outcome and cost-effectiveness of pulsed electromagnetic fields in the treatment of acute scaphoid fractures: a cost-utility analysis. BMC Musculoskelet Disord $16: 84,2015$

23. Huang AJ, Gemperli MP, Bergthold L, Singer SS, Garber A : Health plans' coverage determinations for technology-based interventions: the case of electrical bone growth stimulation. Am J Manag Care 10 : 957-962, 2004

24. Huegel J, Choi DS, Nuss CA, Minnig MCC, Tucker JJ, Kuntz AF, et al. : Effects of pulsed electromagnetic field therapy at different frequencies and durations on rotator cuff tendon-to-bone healing in a rat model. J Shoulder Elbow Surg 27 : 553-560, 2018

25. Jenis LG, An HS, Stein R, Young B : Prospective comparison of the effect of direct current electrical stimulation and pulsed electromagnetic fields on instrumented posterolateral lumbar arthrodesis. J Spinal Disord 13 : 290-296, 2000

26. Kahanovitz $\mathrm{N}$ : Electrical stimulation of spinal fusion: a scientific and clinical update. Spine J 2 : 145-150, 2002

27. Khalifeh JM, Zohny Z, MacEwan M, Stephen M, Johnston W, Gamble P, et al. : Electrical stimulation and bone healing: a review of current technology and clinical applications. IEEE Rev Biomed Eng 11 : 217-232, 2018

28. Linovitz RJ, Pathria M, Bernhardt M, Green D, Law MD, McGuire RA, et al. : Combined magnetic fields accelerate and increase spine fusion: a double-blind, randomized, placebo controlled study. Spine (Phila Pa 1976) 27 : 1383-1389; discussion 1389, 2002

29. Makino T, Tsukazaki H, Ukon Y, Tateiwa D, Yoshikawa H, Kaito T : The biological enhancement of spinal fusion for spinal degenerative disease. Int J Mol Sci 19 : 2430, 2018

30. Markov MS : Expanding use of pulsed electromagnetic field therapies. Electromagn Biol Med 26 : 257-274, 2007

31. Markov MS : Pulsed electromagnetic field therapy history, state of the art and future. Environmentalist $27:$ 465-475, 2007

32. Marks RA : Spine fusion for discogenic low back pain: outcomes in patients treated with or without pulsed electromagnetic field stimulation. Adv Ther 17 : 57-67, 2000

33. Mooney $\vee$ : A randomized double-blind prospective study of the efficacy of pulsed electromagnetic fields for interbody lumbar fusions. Spine (Phila Pa 1976) 15 : 708-712, 1990

34. Morone MA, Feuer $\mathrm{H}$ : The use of electrical stimulation to enhance spinal fusion. Neurosurg Focus 13 : e5, 2002

35. Oishi M, Onesti ST : Electrical bone graft stimulation for spinal fusion: a review. Neurosurgery 47 : 1041-1055; discussion 1055-1056, 2000

36. Orthofix Medical Inc. : CervicalStim spinal fusion therapy. Available at : https://www.orthofix.com/ifus/cervical-stim/

37. Orthofix Medical Inc. : PhysioStim bone healing therapy. Available at : https://www.orthofix.com/ifus/physio-stim/

38. Park SB, Chung CK : Strategies of spinal fusion on osteoporotic spine. J Korean Neurosurg Soc 49 : 317-322, 2011

39. Rikk J, Finn KJ, Liziczai I, Radák Z, Bori Z, Ihász F : Influence of pulsing electromagnetic field therapy on resting blood pressure in aging adults. Electromagn Biol Med 32 : 165-172, 2013

40. Shupak NM, Prato FS, Thomas AW : Therapeutic uses of pulsed magnetic-field exposure: a review. URSI Radio Science Bulletin 2003 : 9-32, 2003

41. Simmons JW Jr, Mooney V, Thacker I : Pseudarthrosis after lumbar spine 
fusion: nonoperative salvage with pulsed electromagnetic fields. Am J Orthop (Belle Mead NJ) $33:$ 27-30, 2004

42. Strauch B, Herman C, Dabb R, Ignarro LJ, Pilla AA : Evidence-based use of pulsed electromagnetic field therapy in clinical plastic surgery. Aesthet Surg J 29 : 135-143, 2009

43. Tian NF, Wu YS, Zhang XL, Mao FM, Xu HZ, Chi YL : Efficacy of electrical stimulation for spinal fusion: a meta-analysis of fusion rate. Spine J 13 : 1238-1243, 2013

44. Victoria $G$, Petrisor B, Drew B, Dick D : Bone stimulation for fracture healing: what's all the fuss? Indian J Orthop 43 : 117-120, 2009

45. Wade $B: A$ review of pulsed electromagnetic field (PEMF) mechanisms at a cellular level: a rationale for clinical use. Am J Health Res 1 : 51 55,2013

46. Waldorff El, Zhang N, Ryaby JT : Pulsed electromagnetic field applica- tions: a corporate perspective. J Orthop Translat 9 : 60-68, 2017

47. Wang C, Liu Y, Wang Y, Wei Z, Suo D, Ning G, et al. : Low-frequency pulsed electromagnetic field promotes functional recovery, reduces inflammation and oxidative stress, and enhances HSP70 expression following spinal cord injury. Mol Med Rep 19 : 1687-1693, 2019

48. Wu N, Lee YC, Segina D, Murray H, Wilcox T, Boulanger $L$ : Economic burden of illness among US patients experiencing fracture nonunion. Orthop Res Rev 5 : 21-33, 2013

49. Zborowski M, Androjna C, Waldorff El, Midura RJ : Comparison of therapeutic magnetic stimulation with electric stimulation of spinal column vertebrae. IEEE Trans Magn 51 : 1-9, 2015

50. Zimmer Biomet : Biomet ${ }^{\circledR}$ EBI bone healing system. Available at : https://www.zimmerbiomet.com/medical-professionals/trauma/product/ biomet-ebi-bone-healing-system.html 